\title{
Chapter 17 \\ Stigma and Confidentiality Indiscretions: \\ Intersecting Obstacles to the Delivery \\ of Pre-Exposure Prophylaxis to Adolescent \\ Girls and Young Women in East Zimbabwe
}

\author{
Morten Skovdal, Phyllis Magoge-Mandizvidza, Rufurwokuda Maswera, \\ Melinda Moyo, Constance Nyamukapa, Ranjeeta Thomas, and \\ Simon Gregson
}

\subsection{Introduction}

At the start of 2019, Zivai's story, a fictitious girl in a relationship with an older man, was discussed by adolescent girls and young women (AGYW) in a series of interviews and focus group discussions. One participant, Jackeline, summarised a core theme running through many of the reflections emerging from this vignette: 'Community norms will make Zivai fail to access Pre-Exposure Prophylaxis (PrEP) because Zivai will be judged if people know that she is using PrEP. In the community there will be several teachings telling them how to behave and not to have sex before marriage. If people find out that Zivai is actually having sex and using prevention methods like PrEP, this is something she will be scolded for. Zivai would have made her life very difficult because health professionals will make sure that they tell Zivai's mother that her daughter is being mischievous and is having sex when the time isn't yet ripe for her to do so.' In this chapter we unpack the fear of AGYW to be associated with, or 'outed' as, someone seeking out or accessing PrEP for HIV prevention, and the implications of this for their utilisation of PrEP.

\footnotetext{
M. Skovdal (西)

Department of Public Health, University of Copenhagen, Copenhagen, Denmark

P. Magoge-Mandizvidza $\cdot$ R. Maswera $\cdot$ M. Moyo

Manicaland Centre for Public Health Research, Biomedical Research and Training Institute,

Harare, Zimbabwe

C. Nyamukapa $\cdot$ S. Gregson

Manicaland Centre for Public Health Research, Biomedical Research and Training Institute,

Harare, Zimbabwe

Department of Infectious Disease Epidemiology, Imperial College London, London, UK

R. Thomas

Department of Health Policy, London School of Economics and Political Science, London, UK
}

(C) The Author(s) 2021

S. Bernays et al. (eds.), Remaking HIV Prevention in the 21st Century, Social

Aspects of HIV 5, https://doi.org/10.1007/978-3-030-69819-5_17 
PrEP is a pill, which, when taken daily, significantly reduces the risk of HIV taking hold and spreading in the body, should it enter the bloodstream. PrEP is efficacious and trials show that PrEP can reduce risk of HIV by over $90 \%$ when taken consistently (McCormack et al. 2016). While PrEP is associated with reductions in HIV acquisition amongst men who have sex with men (MSM) in both high and low-income countries, PrEP trials with African women in the general population have found adherence levels so low, particularly amongst young women, that efficacy could not be ascertained (Marrazzo et al. 2015). Studies suggest that uptake and adherence to PrEP amongst women may be low due to low risk perception and ambivalence around using antiretrovirals for prevention. The FEM-PrEP study, for example, observed that women often underestimated their risk of infection and that perceived risk was associated with greater adherence to PrEP (Corneli et al. 2015).

PrEP is still a relatively new HIV prevention method in many country contexts and settings, including sub-Saharan Africa. Although more than 300,000 people took up PrEP at least once in 2018, this is far short of a global target of 3 million people by 2020 (Joint United Nations Programme on HIV/AIDS (UNAIDS) 2019), and reflects challenges, both to make make PrEP available and accessible, as well as to motivate people 'at risk' to engage with PrEP. While 'correcting' risk perception and addressing ambiguity around availability, usability and effectiveness of PrEP is central to the uptake of PrEP, little has been done to unpack the socio-cultural norms at stake in sub-Saharan Africa, which may prevent AGYW from accessing PrEP.

\subsection{PrEP-Related Stigmas}

The fear of 'being outed' as a PrEP user suggests there are stigmas related to PrEP use. According to Goffman (1963, p. 9), stigma refers to 'the situation of the individual who is disqualified from full social acceptance.' Individuals who are 'disqualified' often inhabit an attribute, which a social context has defined to be devaluing. Individuals who inhabit one or more devalued attributes, may be subject to labelling, stereotyping, status loss and discrimination, depending on the context (Link and Phelan 2001). While stigma in large parts takes place within broader social and structural contexts and inequalities (Parker and Aggleton 2003), how stigma manifests in the lives of discredited people also depends on how they are able to manage stigma. According to Goffman (1963), people adopt strategies to avoid revealing their association with devalued attributes, as has been observed in relation to people's concealing strategies when engaging with and managing ART. We draw on these ideas both to unpack the socio-cultural norms and attitudes that give rise to PrEP-related stigmas, and to demonstrate how declining PrEP is a rational strategy to manage such stigmas.

PrEP-related stigma is however not a new phenomenon. It is present in a small, but expanding number of studies reporting on the experience of men who have sex with men (MSM) in the global North (Eaton et al. 2017; Dubov et al. 2018; Schwartz and Grimm 2019). Only a small number of studies from sub-Saharan Africa allude to 
the potential social risks of taking PrEP. A study in Kenya, for example, explored PrEP use amongst two 'key' population groups: sex workers and MSM. The study found that some participants feared disclosing their PrEP use to family and friends, often out of a concern that daily pill-taking could lead others to think they are HIV positive (Van der Elst et al. 2013). PrEP is an antiretroviral pill, taken daily, and often accessed through HIV services, making it easy to mistake PrEP for antiretroviral therapy, affecting people's engagement with PrEP. Whilst this association did not deter these 'high-risk' individuals from taking PrEP, it did encourage them to carefully manage whom they disclosed their PrEP use to.

PrEP, because of its convenient protection against HIV, is also regularly linked to 'socially unacceptable' behaviour, and is, by some, believed to promote 'risky' sexual behaviour (Knight et al. 2016). A study from the USA has found such stigmatising rhetoric to prevent socially more isolated MSM or transgender women from engaging with PrEP (Mehrotra et al. 2018). In Zimbabwe, a recent study has found that similar perceptions of PrEP meant that young women either declined to engage with PrEP, or opted to discontinue PrEP, fearing their partners would find out and accuse them of extramarital affairs (Gombe et al. 2020). It appears that the decision of some at-risk individuals to reject PrEP is a strategy to manage related stigmas, warranting further attention.

\subsection{The Qualitative Study}

It is our aim to explore (1) the socio-cultural norms that give rise to PrEP-related stigmas; and (2) the implications of these norms and stigmas on AGYWs engagement with PrEP. The study obtained ethical approvals from the Medical Research Council of Zimbabwe (REF: MRCZ/A/2243), the institutional review board of the Biomedical Research and Training Institute in Zimbabwe (REF: AP140/2017), and the Imperial College London Research Ethics Committee (REF: 17IC4160).

\subsubsection{Study Setting and Participants}

The qualitative explorations were conducted in two communities of Manicaland province in east Zimbabwe. The communities were selected for the qualitative study because of their rural/urban location, and for being randomly chosen as intervention sites in the larger study trial. This allowed us to recruit participants with different levels of exposure to PrEP. Half had participated in an intervention introducing them to PrEP (see Thomas et al. 2019), the other half had only heard of PrEP peripherally from other sources. Both communities are characterised by high levels of poverty and HIV. The average HIV prevalence of Manicaland was 11\% in 2015-2016, down from over $25 \%$ at the end of the 1990s (Gregson et al. 2017). 
Thirty AGYW from the two settings were invited to participate in either individual interviews $(n=12)$ or focus group discussions $(F G D)(n=18)$ with the aim of revealing how they encounter, respond to, and negotiate PrEP uptake and engagement. The AGYW were chosen from a baseline survey by purposeful sampling following the criteria that they had to be between the age of 18 and 24, HIV-negative, sexually active, and considered 'at risk' (according to a risk screening tool under development by the World Health Organization (WHO)). Individual interviews with HIV prevention service providers $(n=12)$-recruited from health facilities in the two communities-were also conducted.

\subsubsection{Producing and Making Sense of the Data}

The different methods were applied by a team of experienced qualitative researchers in Shona, the local language of the study area. Interviews and focus group discussions were semi-structured and steered by topic guides. The topic guides for AGYW sought to elicit their experiences and perspectives on HIV risk, relationships, sexuality, HIV prevention practices, services and technologies. The topic guide directed at HIV prevention service providers opened up for a discussion on young people's HIV prevention practices, as well as the opportunities and challenges for young people to access available HIV prevention services and technologies. Interviews and focus groups were digitally recorded, and scheduled to last 60 and $90 \mathrm{~min}$ respectively, a time that was kept and ultimately reflects the length of the interviews.

All interview and focus group recordings were transcribed and translated into English, before being imported into NVivo 12 for thematic coding and interrogation. A cluster of codes, which were deemed relevant for this analysis, were subjected to a thematic network analysis (Attride-Stirling 2001). This involved clustering the codes, or basic themes, into organising themes (or parent nodes in NVivo). We generated three organising themes, providing detail on sexuality stigmas, PrEP stigmas and confidentiality indiscretions. We present each of the organising themes in turn.

\subsection{Findings}

\subsubsection{Sexuality Stigmas: 'A “Child” Shouldn't Be Having Sex'}

Our interviews expose a generational rift when it comes to girls' sexuality. Both AGYW and the healthcare providers spoke about a socio-cultural perception that unmarried AGYW should not be having sex. A number of AGYW said that it was shameful to access HIV prevention methods, 'as they should not be having sex' or 
because 'parents won't accept it because this promotes promiscuity.' Much of the shame was linked to what older people - and their parents in particular-thought about their 'children' being sexually active. However, there was recognition that some parents, whilst struggling to accept their children's sexuality, may also find comfort in knowing that their 'children' are protecting themselves against HIV.

She will be disappointed that I am having sexual relationships before marriage [...] Okay, on one side she might be disappointed and on the other side she will be comforted knowing that I am preserving my life and reducing chances of getting HIV. (Jocelyn, AGYW in interview)

As AGYW consider PrEP they need to weigh up their perceived risk of acquiring HIV with the social risk of their parents passing judgement or being disappointed by their child's decision to engage in pre-marital sex. AGYW are not only the 'children' of their biological parents. They are also 'children' of their community, which means some healthcare providers may pass similar judgement to 'the kids'. One healthcare provider, who, upon reflection, recognises their role in preventing AGYW from accessing PrEP services, illustrates this:

The other thing that demotivates the children to access PrEP services is that when they get to the hospital gate and asks for PrEP, they will be met by someone older, like me, who will judge them for being sexually active at that age, when in fact they should be getting assisted. So yes, health staff can be demotivating. When children come looking for PrEP there is no need to start asking why they are sexually active, just focus on talking them through PrEP. (Healthcare provider (3))

There is a clear tension between how the health system classifies AGYW as adults who do not require guardian permission to access PrEP, and the operational practices, which, shaped by social norms and moralising discourses circulating within the health system, approach AGYW as 'children'. Relatedly, and given the fact that PrEP in this context is being delivered within one place to everyone (at the opportunistic infection clinic), a healthcare provider calls for youth-friendly corners, staffed with personnel not much older than the AGYW themselves.

Middle-aged healthcare providers still have that connotation of fatherhood and motherhood. This should be addressed [...] I think we should have adolescent clinics. I think this will help because the young will go to a place where they can see people of their own age. If they are too old people working there, with the mentality that this client is a child, they will not assist a person as a client. A person who comes for services, should be provided with the services. (Healthcare provider (2))

It is evident from our interviews that there is a fear of AGYW to be seen as sexually active by parents or healthcare providers of a similar age to their parents, which inhibit their engagement with health clinics offering PrEP. PrEP use, however, does not only signal a sexuality, but is associated with a range of other stigmatising attitudes, some of which we will now elucidate. 


\subsubsection{PrEP Stigmas: 'PrEP is for Women with a "Loose Moral"',}

Due to research showing that PrEP might be cost-effective in high-risk populations and less so in the wider population, WHO (2015) developed guidance that PrEP should be offered to population groups with HIV incidence above $3 \%$. This meant that when PrEP was first rolled out in Zimbabwe, as in many other sub-Saharan African countries, it targeted female sex workers. Such messaging and targeting meant that for many of our young female participants, PrEP was considered a prevention method primarily for 'high risk' individuals, including female sex workers, or women with a so-called 'loose moral'. One AGYW stated that if on PrEP, 'they will think I am being mischievous.' Another AGYW drew a direct parallel between being on PrEP and assumed to be a sex worker.

They will be saying things like... let's say it's a woman... her husband occasionally travels or maybe goes to South Africa. Imagine being seen going to get PrEP. The assumption will be that you are a prostitute and you are sleeping around in your husband's absence. (Jocelyn, AGYW in interview)

Another common perception, resonating with observations made elsewhere in Zimbabwe (Gombe et al. 2020), was that PrEP can easily be misunderstood as HIV treatment, demonstrating that residual fears of acquiring HIV still persist. Because PrEP is an antiretroviral drug, accessed through sexual health clinics, just like antiretroviral drugs for HIV treatment, a number of AGYW spoke about their fear of being seen as HIV positive and on treatment.

People are scared of taking PrEP because the tablets look like the ones that are taken by HIV positive people so one may be scared of what people will say about the person taking tablets that are similar to that. (Alice, AGYW in interview)

Healthcare workers noted similar concerns, and offered insights to alternative ways of delivering PrEP.

PrEP, should be dispensed in another container. They don't want it to be dispensed in its original containers which are easily identified. (Healthcare providers (W1))

The stigmatising attitudes highlighted above underline the importance of PrEP delivery discretion.

\subsubsection{Confidentiality Indiscretions: 'The Issue of Confidentiality is Their Greatest Worry'}

AGYW worry about walking into clinic spaces where PrEP is on offer. Many of our young female participants said that they would be 'shy to go to health facilities to look for these methods'-worrying about being seen or heard seeking out PrEP services, or, as noted above, mistaken as someone on HIV treatment. These concerns 
indicate that many AGYW do not feel that community-embedded clinics, although accessible because of their proximity, can ensure confidentiality. Both healthcare workers and the young women themselves noted a fear amongst AGYW to run into neighbours or community members at the clinic, believing they may inform their parents or partners about their clinic visit.

They [AGYW] do not want to go to the clinic, particularly when it is busy. They always feel that there will be someone who can recognize them and report them to their parents [...] I don't know what we can do because some say when they get to the hospital they will be seen by neighbours. (Healthcare provider (3))

A girl will feel shy or embarrassed to be seen by community members or other close people accessing these methods from the local clinic. She will also fear for her partner knowing that she is using PrEP or condoms, which leads to her being dumped by her partner because of that. (Mercy, AGYW in FGD)

The participants not only noted that confidentiality is hard to maintain in public clinic spaces, but also alluded to AGYW's lack of trust in the ability of healthcare providers to maintain confidentiality. Like Jackeline, a number of participants believed there was a chance that some healthcare providers may disclose details of AGYW's use of, or interest in, PrEP to relatives or acquaintances in the community. Mary stressed the importance of healthcare providers maintaining confidentiality.

Upholding privacy is important, especially to the nurses who would have served someone who has visited the clinic to access HIV prevention methods. They should be told that it is not allowed for them to go and spread the word that so and so has come to the clinic to access PrEP or condoms or getting tested. (Mary, AGYW in FGD)

Healthcare providers recognised this concern and went to great lengths in the interviews to explain their efforts to ensure that the young women felt safe and comfortable to open up about their sexual risk practices and need for PrEP.

We are trying by all means to offer services in a cheerful manner so that the patient feels free to open up. We give them a platform, a confidential space, for whatever discussion we are going to have; it will be between the two of us and no-one else will hear about what we have discussed. So maybe that will give the patient confidence in us that anyone in the street will not know what we are talking about. (Healthcare provider (2))

Our findings suggest that AGYW and healthcare providers are acutely aware of the community-embeddedness of local clinics delivering PrEP. This embeddedness, coupled with the stigmas associated with PrEP, heightens the social risk of confidentiality indiscretions. The decision of AGYW not to go to the clinic during busy times, or all together, or not to disclose details about at-risk behaviours to healthcare providers is a strategic one. A conscious choice of 'information management' (Goffman 1963) to minimise their association with PrEP and related stigmas. 


\subsection{Discussion}

Our study reveals two prevailing socio-cultural norms around girls' sexuality and use of HIV prevention methods. One, a norm that girls should not be having sex before marriage; two, a norm that uptake of PrEP signals promiscuity. According to our participants, girls who have sex before marriage, or make use of PrEP may be subject to stigmatising attitudes and rhetoric. Out of fear of being associated with these stigmas, heightened by concerns of clinic-level confidentiality indiscretions, AGYW, as a way of managing stigma, may actively avoid seeking out local PrEP services. Stigma and the worry of AGYW that privacy and confidentiality cannot be maintained in local health clinics and by local healthcare providers, presents a major barrier to the uptake of PrEP.

These findings are constrained by some methodological limitations. First, the study relies on self-reported data. Future research may consider adopting a more in-depth and longitudinal ethnographic approach. Second, our study was crosssectional and only provides a brief snapshot of perspectives at a particular moment in time. Third, whilst our rural and urban findings were broadly similar, the generalisability of our findings is limited and may not apply to other country settings, as the socio-cultural norms and PrEP service delivery structures characteristic of our setting may vary from others. This study only reports on the perspectives of AGYW and healthcare providers. In future studies we will broaden the scope and include the perspectives and experiences of parents, community members and young men.

Nonetheless, our findings resonate with observations made elsewhere. Public discourses of PrEP as a 'promiscuity pill' have also been noted amongst gay men in north America (Eaton et al. 2017) and in Malaysia (Bourne et al. 2017), with implications for their interest in adopting PrEP. Our observation that potential PrEP users express a fear of stigma by healthcare providers, because of their socio-cultural values and norms, has been noted elsewhere. In San Francisco, USA, many men on PrEP have reported feeling stigmatised by healthcare providers (Liu et al. 2014), and accounts from female sex workers in South Africa stress the importance of health clinics being safe spaces, free of judgement from healthcare providers (Eakle et al. 2018). Resonating with an argument made by Müller et al. (2016) in relation to sexual and reproductive health policies and services for adolescents in South Africa, our observations suggest that front-line healthcare providers, by virtue of how their own moral compasses and values influence their delivery of PrEP, act as gatekeepers for the implementation of PrEP policies and services. Müller et al. (2016) go on to argue that nurse morals and judgments can result in uneven policy translations and service provisions, jeopardising sexual and reproductive health services for adolescents.

Our findings are not wholly unexpected. 'Not unexpected' because our observations resonate with earlier and broader discussions of adolescents' uptake of sexual and reproductive health services (Bearinger et al. 2007), and reflect long-standing challenges pertaining to family planning (Tavrow et al. 2012), condom use (MacPhail and Campbell 2001) and treatment of sexually transmitted infections 
(Sales et al. 2007). It is disappointing that we do not seem to have managed to learn very much from the plethora of evidence concerning social and structural approaches to HIV prevention (Campbell et al. 2005; Gupta et al. 2008). The enthusiasm to harness recent biomedical and health service successes in HIV treatment and expand PrEP to AGYW is understandable. Not least because PrEP is considered costeffective in averting HIV infection (Schackman and Eggman 2012) and because it offers women the opportunity to take control over sexual risks. However, efforts to scale up PrEP must build on decades of social science research that have scrutinised the intersections between socio-cultural norms of youth sexuality, stigma and engagement with a variety of sexual and reproductive health services.

For instance, there have been numerous calls for 'youth-friendly' sexual and reproductive health services (Kennedy et al. 2013). It is evident from our findings that PrEP also needs to be delivered in youth friendly corners, where (young) health care providers are trained to welcome and engage young people in ways that foster trustful relations and make young people feel they are in a safe space. Mmari and Magnani (2003) have found youth-friendly corners to increase sexual and reproductive health services uptake in Zambia, but also noted that health services do not operate in a vacuum, and addressing socio-cultural norms and barriers at a community-level, may have an even greater impact. In an action research project in Kenya, Chubb (2018) innovatively explores the role of traditional meeting and dialogue structures (like the Kenyan mabaraza) for intergenerational dialogues on sex-related issues. She concludes that 'community conversations' about young people's sexuality, introduced in, and supported by, traditional structure, are key for breaking down community-level barriers to young people's sexual health.

While most of our observations reflect long-standing challenges to young people's engagement with sexual and reproductive health services, PrEP-specific factors hindering uptake did emerge. More broadly, it appears that PrEP, and how the technology was historically introduced to MSM and female sex workers, is shaping the public imagination about PrEP in a way that might be quite distinctive. Our findings suggest that PrEP for AGYW in the general population, it is saddled with an additional layer of social resistance. This resistance requires health service providers to carefully consider how best to deliver PrEP, for instance through youth-friendly corners, and re-thinking how PrEP pills are packed and handed over to AGYW. The ' $\mathrm{V}$ ' initiative in southern Africa is looking to address this challenge by repackaging PrEP tablets in containers that look like lip-gloss (www.conrad.org/launchingv). More broadly however, it also calls for critical reflection on how PrEP has become an intervention for AGYW in sub-Saharan Africa and the implications of this for AGYW. 


\subsection{Conclusions}

The biomedical nature of PrEP heightens the impact of socio-cultural norms as they relate to adult attitudes to HIV prevention methods and sex. PrEP may provide AGYW with control over their sexual risks, but the journey to access PrEP involves a series of social risks, which appear to outweigh the perceived benefits of engaging with PrEP amongst AGYW in eastern Zimbabwe. To shift this balance there is an urgent need to tackle the range of socio-cultural norms and social practices that interact to form the context that prevents AGYW from taking advantage of PrEP. We have previously noted that approaching engagement with PrEP as a social practice is key to identifying and responding to the combination of social and structural interventions needed to make engagement with PrEP a possible and desirable thing to do for AGYW (Skovdal 2019).

Acknowledgments We would like to thank all the participants for their time and valuable insight. We would also like to thank Rangarirayi Primrose Nyamwanza, Douglas Muchemwa, Rumbidzai Bangidza and Moreblessing Mangwiro for research support. The study was supported by the National Institute of Mental Health (NIMH) (Grant R01MH114562-01) and the Bill and Melinda Gates Foundation (BMGF) (OPP1161471). The content is solely the responsibility of the authors and does not necessarily represent the official views of the NIMH or the BMGF. CN and SG acknowledge joint MRC Centre for Global Infectious Disease Analysis funding from the UK Medical Research Council and Department for International Development (MR/R015600/1).

\section{References}

Attride-Stirling, J. (2001). Thematic networks: An analytic tool for qualitative research. Qualitative Research, 1(3), 385-405. https://doi.org/10.1177/146879410100100307.

Bearinger, L. H., Sieving, R. E., Ferguson, J., \& Sharma, V. (2007). Global perspectives on the sexual and reproductive health of adolescents: Patterns, prevention, and potential. The Lancet, 369(9568), 1220-1231. https://doi.org/10.1016/S0140-6736(07)60367-5.

Bourne, A., Cassolato, M., Thuan Wei, C. K., Wang, B., Pang, J., Lim, S. H., et al. (2017). Willingness to use pre-exposure prophylaxis (PrEP) for HIV prevention among men who have sex with men (MSM) in Malaysia: Findings from a qualitative study. Journal of the International AIDS Society, 20(1), 21899.

Campbell, C., Foulis, C., Maimane, S., \& Sibiya, Z. (2005). The impact of social environments on the effectiveness of youth HIV-prevention: A south African case study. AIDS Care, 17(4), 471-478.

Chubb, L. (2018). Struggle for a sustainable solution: Building safe sex-talk spaces with a rural kenyan community. $\mathrm{PhD}$ thesis, Auckland: The University of Auckland. Available from: https:// researchspace.auckland.ac.nz/handle/2292/44012

Corneli, A., Perry, B., Agot, K., Ahmed, K., Malamatsho, F., \& Van Damme, L. (2015). Facilitators of adherence to the study pill in the FEM-PrEP clinical trial. PLoS One, 10(4), e0125458e0125458. https://doi.org/10.1371/journal.pone.0125458.

Dubov, A., Galbo, P., Jr., Altice, F. L., \& Fraenkel, L. (2018). Stigma and shame experiences by MSM who take PrEP for HIV prevention: A qualitative study. American Journal of Men's Health, 12(6), 1843-1854. 
Eakle, R., Bourne, A., Mbogua, J., Mutanha, N., \& Rees, H. (2018). Exploring acceptability of oral PrEP prior to implementation among female sex workers in South Africa. Journal of the International AIDS Society, 21(2), e25081.

Eaton, L. A., Kalichman, S. C., Price, D., Finneran, S., Allen, A., \& Maksut, J. (2017). Stigma and conspiracy beliefs related to pre-exposure prophylaxis (PrEP) and interest in using PrEP among black and white men and transgender women who have sex with men. AIDS and Behavior, 21 (5), 1236-1246.

Goffman, E. (1963). Stigma: Notes on the management of spoiled identity. Englewood Cliffs, NJ: Prentice-Hall.

Gombe, M. M., Cakouros, B. E., Ncube, G., Zwangobani, N., Mareke, P., Mkwamba, A., et al. (2020). Key barriers and enablers associated with uptake and continuation of oral pre-exposure prophylaxis (PrEP) in the public sector in Zimbabwe: Qualitative perspectives of general population clients at high risk for HIV. PLoS One, 15(1), e0227632. https://doi.org/10.1371/ journal.pone.0227632.

Gregson, S., Mugurungi, O., Eaton, J., Takaruza, A., Rhead, R., Maswera, R., et al. (2017). Documenting and explaining the HIV decline in East Zimbabwe: The Manicaland general population cohort. BMJ Open, 7(10), e015898.

Gupta, G. R., Parkhurst, J. O., Ogden, J. A., Aggleton, P., \& Mahal, A. (2008). Structural approaches to HIV prevention. The Lancet, 372(9640), 764-775.

Kennedy, E. C., Bulu, S., Harris, J., Humphreys, D., Malverus, J., \& Gray, N. J. (2013). "Be kind to young people so they feel at home": A qualitative study of adolescents' and service providers' perceptions of youth-friendly sexual and reproductive health services in Vanuatu. BMC Health Services Research, 13(1), 455.

Knight, R., Small, W., Carson, A., \& Shoveller, J. (2016). Complex and conflicting social norms: Implications for implementation of future HIV pre-exposure prophylaxis (PrEP) interventions in Vancouver, Canada. PLoS One, 11(1), e0146513.

Link, B. G., \& Phelan, J. C. (2001). Conceptualizing stigma. Annual Review of Sociology, 27, 363-385. https://doi.org/10.1146/annurev.soc.27.1.363.

Liu, A., Cohen, S., Follansbee, S., Cohan, D., Weber, S., Sachdev, D., et al. (2014). Early experiences implementing pre-exposure prophylaxis (PrEP) for HIV prevention in San Francisco. PLoS Medicine, 11(3), e1001613.

MacPhail, C., \& Campbell, C. (2001). 'I think condoms are good but, aai, I hate those things': Condom use among adolescents and young people in a southern African township. Social Science \& Medicine, 52(11), 1613-1627. https://doi.org/10.1016/s0277-9536(00)00272-0.

McCormack, S., Dunn, D. T., Desai, M., Dolling, D. I., Gafos, M., Gilson, R., et al. (2016). Pre-exposure prophylaxis to prevent the acquisition of HIV-1 infection (PROUD): Effectiveness results from the pilot phase of a pragmatic open-label randomised trial. Lancet, 387(10013), 53-60. https://doi.org/10.1016/S0140-6736(15)00056-2.

Marrazzo, J. M., Ramjee, G., Richardson, B. A., Gomez, K., Mgodi, N., Nair, G., et al. (2015). Tenofovir-based preexposure prophylaxis for HIV infection among African women. New England Journal of Medicine, 372(6), 509-518.

Mehrotra, M. L., Amico, K. R., McMahan, V., Glidden, D. V., Defechereux, P., Guanira, J. V., et al. (2018). The role of social relationships in PrEP uptake and use among transgender women and men who have sex with men. AIDS and Behavior, 22(11), 3673-3680.

Mmari, K. N., \& Magnani, R. J. (2003). Does making clinic-based reproductive health services more youth-friendly increase service use by adolescents? Evidence from Lusaka, Zambia. Journal of Adolescent Health, 33(4), 259-270. https://doi.org/10.1016/S1054-139X(03) 00062-4.

Müller, A., Röhrs, S., Hoffman-Wanderer, Y., \& Moult, K. (2016). "You have to make a judgment call" - Morals, judgments and the provision of quality sexual and reproductive health services for adolescents in South Africa. Social Science \& Medicine, 148, 71-78. https://doi.org/10. 1016/j.socscimed.2015.11.048.

Parker, R., \& Aggleton, P. (2003). HIV and AIDS-related stigma and discrimination: A conceptual framework and implications for action. Social Science \& Medicine, 57(1), 13-24. 
Sales, J. M., DiClemente, R. J., Rose, E. S., Wingood, G. M., Klein, J. D., \& Woods, E. R. (2007). Relationship of STD-related shame and stigma to female adolescents' condom-protected intercourse. Journal of Adolescent Health, 40(6), 573.e571-573.e576. https://doi.org/10.1016/j. jadohealth.2007.01.007.

Schackman, B. R., \& Eggman, A. A. (2012). Cost-effectiveness of pre-exposure prophylaxis for HIV: A review. Current Opinion in HIV/AIDS, 7(6), 587-592.

Schwartz, J., \& Grimm, J. (2019). Stigma communication surrounding PrEP: The experiences of a sample of men who have sex with men. Health Communication, 34(1), 84-90. https://doi.org/ 10.1080/10410236.2017.1384430.

Skovdal, M. (2019). Facilitating engagement with PrEP and other HIV prevention technologies through practice-based combination prevention. Journal of the International AIDS Society, 22 (S4), e25294. https://doi.org/10.1002/jia2.25294.

Tavrow, P., Withers, M., \& McMullen, K. (2012). Age matters: Differential impact of service quality on contraceptive uptake among post-abortion clients in Kenya. Culture, Health and Sexuality, 14(8), 849-862.

Thomas, R., Skovdal, M., Galizzi, M., Schaefer, R., Moorhouse, L., Nyamukapa, C., et al. (2019). Improving risk perception and uptake of pre-exposure prophylaxis (PrEP) through interactive feedback-based counselling with and without community engagement in young women in Manicaland, East Zimbabwe: Study protocol for a pilot randomised trial. Trials, 20, 668. https://doi.org/10.1186/s13063-019-3791-8.

UNAIDS. (2019). Global AIDS update 2019 - Communities at the Centre. Geneva: Joint United Nations Programme on HIV/AIDS.

Van der Elst, E. M., Mbogua, J., Operario, D., Mutua, G., Kuo, C., Mugo, P., et al. (2013). High acceptability of HIV pre-exposure prophylaxis but challenges in adherence and use: Qualitative insights from a phase I trial of intermittent and daily PrEP in at-risk populations in Kenya. AIDS and Behavior, 17(6), 2162-2172. https://doi.org/10.1007/s10461-012-0317-8.

WHO. (2015). Guideline on when to start antiretroviral therapy and on pre-exposure prophylaxis for HIV. https://www.who.int/hiv/pub/guidelines/earlyrelease-arv/en/. Accessed 7 January 2019.

Open Access This chapter is licensed under the terms of the Creative Commons Attribution 4.0 International License (http://creativecommons.org/licenses/by/4.0/), which permits use, sharing, adaptation, distribution and reproduction in any medium or format, as long as you give appropriate credit to the original author(s) and the source, provide a link to the Creative Commons license and indicate if changes were made.

The images or other third party material in this chapter are included in the chapter's Creative Commons license, unless indicated otherwise in a credit line to the material. If material is not included in the chapter's Creative Commons license and your intended use is not permitted by statutory regulation or exceeds the permitted use, you will need to obtain permission directly from the copyright holder.

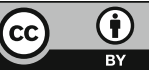

